\title{
An Equation of State for Partially Ionized Plasmas: The Coulomb Contribution to the Free Energy
}

\author{
D.P. Kilcrease ${ }^{a, *}$, J. Colgan ${ }^{a}$, P. Hakel ${ }^{b}$, C.J. Fontes ${ }^{b}$, M.E. Sherrill ${ }^{a}$ \\ ${ }^{a}$ Theoretical Division, Los Alamos National Laboratory, Los Alamos, NM 87545, USA \\ ${ }^{b}$ Computational Physics Division, Los Alamos National Laboratory, Los Alamos, NM \\ 87545, USA
}

\begin{abstract}
We have previously developed an equation of state (EOS) model called ChemEOS (Hakel and Kilcrease, Atomic Processes in Plasmas, Eds., J. Cohen et al., AIP, 2004) for a plasma of interacting ions, atoms and electrons. It is based on a chemical picture of the plasma and is derived from an expression for the Helmholtz free energy of the interacting species. All other equilibrium thermodynamic quantities are then obtained by minimizing this free energy subject to constraints, thus leading to a thermodynamically consistent EOS. The contribution to this free energy from the Coulomb interactions among the particles is treated using the method of Chabrier and Potekhin (Phys. Rev. E 58, 4941 (1998)) which we have adapted for partially ionized plasmas. This treatment is further examined and is found to give rise to unphysical behavior for various elements at certain values of the density and temperature where the Coulomb coupling begins to become significant and the atoms are partially ionized. We examine the source of this unphysical behavior and suggest corrections that produce acceptable results. The sensitivity of the thermodynamic properties and frequency-dependent opacity of iron is examined with and without these corrections. The corrected EOS is used to determine the fractional ion populations and level populations for a new generation of OPLIB low-Z opacity tables currently being prepared at Los Alamos National Laboratory with the ATOMIC code.
\end{abstract}

Keywords: opacity, equation of state

\footnotetext{
* Corresponding author

Email address: dpk@lanl.gov (D.P. Kilcrease)
}

Preprint submitted to High Energy Density Physics

May 28, 2015 


\section{Introduction}

The cornerstone of any opacity calculation is the equation of state that provides the populations of the occupied states of the atoms and ions that make up the system under consideration. The Los Alamos opacity code ATOMIC [2, 3] uses the ChemEOS equation of state (EOS) model [1] for this purpose. ChemEOS is a chemical picture equation of state based on a numerical minimization of the Helmholtz free energy. Since the starting point is the free energy, all subsequently derived thermodynamic quantities will be thermodynamically consistent. This free energy model is based on the assumption of the separability of the system Hamiltonian into components thus leading to the additivity of the free energies for these components. In particular we have for the ChemEOS total Helmholtz free energy of the system

$$
F=F_{e}+F_{i}+F_{i n t}+F_{H S}+F_{C},
$$

where $F_{e}$ and $F_{i}$ are respectively the electron and ion components of the kinetic energy, and $F_{\text {int }}$ is due to the internal energy partition function of the atoms and ions with bound electrons. These three terms constitute the ideal Saha-Boltzmann model. Non-ideal effects are taken into account by incorporating occupation probabilities in $F_{\text {int }}$, the addition of a hard sphere contribution $F_{H S}$ that takes the size of the atoms and ions into account, and the Coulomb interaction term $F_{C}$ for the electrostatic interactions among the electrons and ions. The Coulomb interaction term is that proposed by Chabrier et al. [4]. It consists of three parts

$$
F_{C}=F_{i i}+F_{e e}+F_{i e},
$$

where $F_{i i}$ refers to a one component ion plasma, $F_{e e}$ refers to a one component quantum electron plasma, and $F_{i e}$ is an electron-ion interaction term. The electron-ion interaction term has been constructed so that it has the appropriate limit in the weak and strong coupling regimes, i.e.

$$
f_{i e}=\frac{F_{i e}}{k T N_{i}}=\frac{-\Gamma_{e}\left[c_{D H} \sqrt{\Gamma_{e}}+c_{T F} a \Gamma_{e}^{\nu} g_{1} h_{1}\right]}{1+\left(b \sqrt{\Gamma_{e}}+a g_{2} \Gamma_{e}^{\nu} / r_{s}\right) h_{2}}
$$

In Eq. 3, $N_{i}$ is the number of ions, $k T$ is the temperature in energy units, $\Gamma_{e}$ is the electron coupling constant, $r_{s}$ is the electron density parameter, $c_{T F}$ is the zero temperature Thomas-Fermi strong coupling contribution, and $a, b$, 
$g_{1}, g_{2}, h_{1}, h_{2}$, and $\nu$ are fitting parameters defined in Ref. [4] and given in the appendix below along with the definition of other fundamental quantities. In the weak coupling regime the $f_{i e}$ term approaches the weak coupling DebyeHückel limit for interacting electrons and ions given by $c_{D H}$ which is,

$$
c_{D H}=\frac{Z}{\sqrt{3}}\left[(1+Z)^{3 / 2}-1-Z^{3 / 2}\right],
$$

where $Z$ is the ion charge. The term $-1-Z^{3 / 2}$ serves to cancel the weak coupling parts of $F_{e e}$ and $F_{i i}$ since these are not separable from the electronion interaction in this limit and are included in the Debye-Hückel limit of $f_{i e}$. If there is a mixture of ions of different charges, an effective charge must be used. Our adopted model for selecting this effective charge will be discussed below in Section 3.

\section{Modifications for partially ionized plasmas}

The formulation of the electron-ion interaction free energy term, $F_{i e}$, in the ChemEOS equation of state follows the work of Chabrier et al. [4] for fully ionized plasmas, which we have adapted to mixtures of ions. It is expressed as a smooth interpolation between the weak coupling Debye-Hückel limit for point charges and the strong coupling Thomas-Fermi limit. This term does not take into account the possibility of electrons becoming bound by the ions as occurs in partially ionized plasmas. Unmodified, this formalism may lead to unphysical features when applied to partially ionized plasmas. In Figure 1 we show the result of using the unmodified $F_{C}$ approximation on the average ionization of iron as a function of increasing density for various isotherms. Unphysical features are present representing pressure ionization followed by recombination as the density increases. As a result of this behavior, unphysical features are also present in the pressure as seen in Figure 2. We examined which of the different contributions to the free energy was responsible for causing these unphysical effects and confirmed that the electron-ion interaction term $F_{i e}$ was the largest contributor. A shortcoming of the fully ionized plasma $F_{i e}$ model described above is that it includes attractive forces but does not account for electron-ion binding when these forces are sufficiently strong. In fact, in our model these bound state effects are already taken into account quantum mechanically in the internal free energy term $F_{\text {int }}$. As a partial correction to an overly strong $F_{i e}$ interaction term, we modify the Debye-Hückel component $c_{D H}$ to take into account a 
radius of closest approach between the charged particles. The Debye-Hückel theory can be reformulated for interacting particles of finite radius according to Ref. [9]. If we then choose this radius of closest approach $r_{c}$ to be the radius from the ion where the electron's kinetic energy $\bar{E}_{K E}$ is equal to the electron's potential energy in the field of the ion (i.e. $\bar{E}_{K E}=Z e^{2} / r_{c}$ ), we arrive at the following modified Debye-Hückel component $[6,8]$

$$
c_{D H}=\frac{Z}{\sqrt{3}}\left[(1+Z)^{3 / 2} \tau(x)-1-Z^{3 / 2}\right]
$$

where

$$
\tau(x)=\frac{3}{x^{3}}\left[\ln (1+x)-x+\frac{1}{2} x^{2}\right]
$$

and

$$
x=\frac{r_{c}}{\lambda_{D}}=\frac{4}{3}\left(\frac{e}{\sqrt{k T}}\right)^{3} \sqrt{\pi\left(\sum_{s \neq e} n_{s} Z_{s}^{2}+n_{e} \Theta_{e}(\eta)\right)} \frac{\sum_{s \neq e} n_{s} Z_{s}}{\sum_{s \neq e} n_{s}} \frac{I_{1 / 2}(\eta)}{I_{3 / 2}(\eta)} .
$$

Here $e$ is the unit electric charge, $n_{s}$ is the number density of ion $s, n_{e}$ is the electron number density, and $Z_{s}$ is the charge of ion $s$. The DebyeHückel screening length for a mixture of ion charges and electrons is $\lambda_{D}$ (see appendix) and the electron degeneracy factor is

$$
\Theta_{e}(\eta)=\frac{I_{-1 / 2}(\eta)}{I_{1 / 2}(\eta)}
$$

The Fermi integrals are given by

$$
I_{\nu}(\eta)=\frac{1}{\Gamma(\nu+1)} \int_{0}^{\infty} \frac{t^{\nu} d t}{e^{t-\eta}+1} .
$$

The normalization of the Fermi integral has been chosen so that $\partial I_{\nu}(\eta) / \partial \eta=$ $I_{\nu-1}(\eta)$, and for non-degenerate electrons all $I_{\nu}(\eta) \rightarrow 1$. The electron degeneracy parameter is $\eta=\mu_{e} / k T$ where $\mu_{e}$ is the ideal electron gas chemical potential. For low densities and high temperatures where $\lambda_{D}>>r_{c}$, the multiplicative factor $\tau$ behaves as $\tau(x) \rightarrow 1$ as $x \rightarrow 0$, otherwise $0 \leq \tau(x)<1$.

Our approximation to the electron-ion interaction free energy $f_{i e}$ is subject to some limitations. Shortcomings inherent in the Debye-Hückel interaction with the radius of closest approach modification are discussed by Harris 
et al. [6]. Additionally, the original $f_{i e}$ formulation of Chabrier et al. [4] does not include electron degeneracy and this shortcoming is only partly ameliorated by the inclusion of these effects in the radius of closest approach. By far, the largest effect of electron degeneracy is in the electron kinetic energy contribution to the free energy $F_{e}$ (Eq. 1), which our model treats correctly to all orders in the electron degeneracy parameter $\eta$. Although we have made corrections to the weak coupling limit of $f_{i e}$ arising from electron-ion bound state formation, we have not made any alteration for this effect to the strong coupling limit. Therefore, our treatment for bound state effects on $f_{i e}$ is only approximate.

The modification of the electron-ion interaction term $f_{i e}$ produces corresponding changes to the electron-ion contributions to the pressure and energy calculated from the partial derivatives with respect to volume $V$ and temperature respectively. For the pressure

$$
P_{i e}=\frac{k T N_{i}}{3 V}\left\{\frac{\partial f_{i e}}{\partial \Gamma_{e}} \Gamma_{e}-\frac{\partial f_{i e}}{\partial r_{s}} r_{s}+\frac{\partial f_{i e}}{\partial x_{r}} x_{r}-3 \frac{\partial f_{i e}}{\partial \tau} \frac{d \tau}{d x} V \frac{\partial x}{\partial V}\right\},
$$

and for the energy

$$
E_{i e}=k T N_{i}\left\{\frac{\partial f_{i e}}{\partial \Gamma_{e}} \Gamma_{e}-\frac{\partial f_{i e}}{\partial \tau} \frac{d \tau}{d x} T \frac{\partial x}{\partial T}\right\} .
$$

The additional new terms are

$$
\begin{gathered}
\frac{\partial f_{i e}}{\partial \tau}=-\frac{\Gamma_{e}^{3 / 2}}{1+\left[b \sqrt{\Gamma_{e}}+a g_{2} \Gamma_{e}^{\nu} / r_{s}\right] h_{2}} \frac{Z}{\sqrt{3}}(1+Z)^{3 / 2} \\
\frac{d \tau}{d x}=-\frac{3}{x}\left(\tau(x)-\frac{1}{1+x}\right)
\end{gathered}
$$

The partial derivatives $\partial x / \partial T, \partial x / \partial V$ are as given by Däppen et al. [8] (their equations A47, A48, A29, A30), while the partial derivatives $\partial f_{i e} / \partial \Gamma_{e}$, $\partial f_{i e} / \partial r_{s}$, and $\partial f_{i e} / \partial x_{r}$ are obtained by straightforward differentiation of our Eq. 3 modified by Eq. 5 .

\section{Effective parameters of the partially ionized system}

In order to use the Coulomb interaction free energies of Chabrier et al. [4] we need to devise some effective ion charge and coupling constants to replace 
those of a fully ionized plasma in the Coulomb contributions $F_{i i}, F_{e e}$, and $F_{i e}$. We have chosen the following simple prescription. The electron number density $n_{e}^{*}$, ion number density $n_{i}^{*}$, and average ionization $Z^{*}$ of this fictitious fully ionized system are determined by demanding that fit functions in Eq. 2 recover the Debye-Hückel limit at low densities; hence we set

$$
n_{e}^{*}+n_{i}^{*} Z^{* 2}=n_{e}+\sum_{s \neq e} n_{s} Z_{s}^{2}
$$

with

$$
n_{e}^{*}=Z^{*} n_{i}^{*}
$$

The simplest way of closing the above system of two equations for three unknowns is to select $n_{e}^{*}=n_{e}$, which yields

$$
Z^{*}=\frac{1}{n_{e}} \sum_{s \neq e} n_{s} Z_{s}^{2},
$$

and $n_{i}^{*}$ is then given by Eq. 15. In the limit of approaching a neutral system $\left(n_{e} \rightarrow 0\right)$, all free electrons come from the singly-charged ion $\left(n_{e} \approx n_{s}\right.$ for $\left.z_{s}=1\right)$, so in this case Eq. 16 becomes

$$
\lim _{n_{e} \rightarrow 0} Z^{*}=1
$$

Effective electron and ion-coupling parameters are linked by the usual relation valid for fully ionized systems, i.e.

$$
\Gamma_{i}^{*}=\Gamma_{e} Z^{* 5 / 3}
$$

Since $\Gamma_{e}$ depends only on $n_{e}$ and $k T$ it remains unchanged. Also unchanged are $r_{s}$ and $x_{r}$ which depend only on $n_{e}$ (see appendix).

This approach reproduces both the Debye-Hückel excess free energy at low densities as well as the fully (pressure) ionized system at high densities. In the intermediate region of partial ionization with strong coupling this procedure provides a smooth transition between these two well-characterized limits. Other methods have been suggested to choose an effective ion charge and coupling constants [5] for these Coulomb interaction terms. 


\section{Results}

The EOS modifications described in Section 2 produce significant changes to the plasma average ionization in the pressure ionization regime and the adjacent recombination region of the density temperature space of our opacity tables. These changes are particularly noticeable in the third and fourth row elements of the periodic table. As an example, we examine the results of the above changes to the EOS model in ATOMIC for an iron plasma. Figure 1 shows the average ionization of iron as a function of mass density for a range of isotherms from $30 \mathrm{eV}$ to $300 \mathrm{eV}$. The average ionization is shown without and with the EOS changes. As can be seen, the original isotherms show unphysical significant recombination after pressure ionization as the density increases from 0.1 to $1000 \mathrm{~g} \mathrm{~cm}^{-3}$. The problems manifest in the average ionization are also present in other thermodynamic quantities such as the total energy and pressure. Figure 2 shows the unphysical effects on the total pressure leading to violation of the mechanical stability requirement $(\partial P / \partial \rho)_{T,\left\{N_{i}\right\}}>0[11]$. We plot the absolute value of the Coulomb contribution to the total pressure $\left|P_{C}\right|$ (since it is negative) from the uncorrected EOS showing a large increase in its magnitude in the neighborhood of the unphysical region of the total pressure. After the correction is applied the absolute value of the Coulomb contribution is decreased leading to a total pressure curve that is now mechanically stable.

The recent iron opacity experiment at Sandia National Laboratory reported by Bailey et al. [12] shows some significant disagreements between theoretical calculations and experiment. We examine the impact of the EOS modifications discussed above on our theoretical spectral simulation of this experiment using ATOMIC $[2,3]$. The decrease in the intensity of the principal quantum number $n=2$ to 3 line transitions observed in our ATOMIC calculation resulting from changes to the EOS is due to the shift in the ionization balance from $\bar{Z}=16.5$ to 15.5 as seen in Figure 3 . This shift to a slightly less average ionization is consistent with the changes in average ionization found in Figure 1 and is due to our approximate correction for the effect of bound states on the electron-ion contribution to the free energy. The 2 to 3 line transitions shown in Figure 4 are due primarily to the $2 \mathrm{p}$ $3 \mathrm{~d}$ lines in N-like and $2 \mathrm{~s}-3 \mathrm{p}$ lines in F-like ions. The decrease is compensated for by 2 to 3 transition lines in Na-like ions (and similar) that have absorption features at longer wavelengths than are shown in the figure (13.5 to 16 Angstroms). However the average population of the $\mathrm{L}$ shell increases 


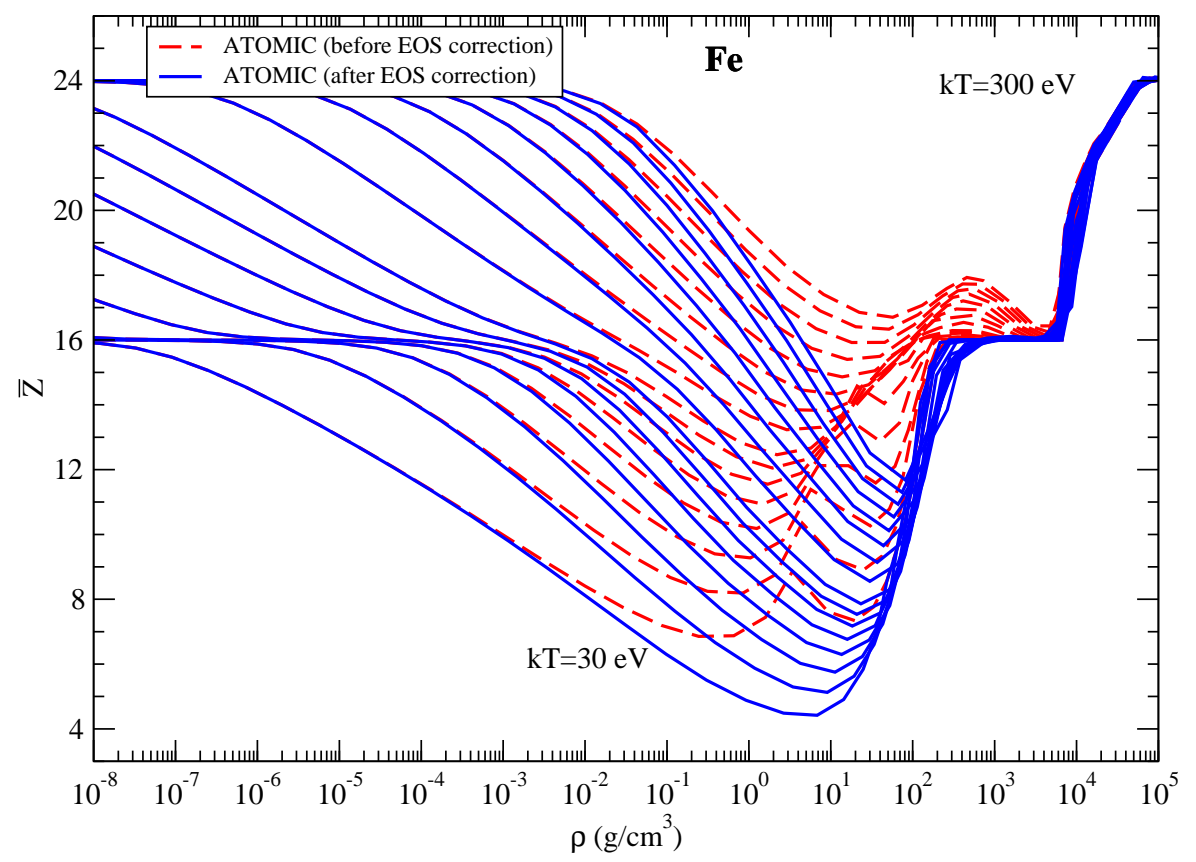

Figure 1: Average ionization of iron as a function of density for a range of isotherms showing unphysical behavior for the uncorrected EOS (red) and more reasonable behavior for the corrected EOS (blue). The isotherms span the 30 to $300 \mathrm{eV}$ temperature range. 


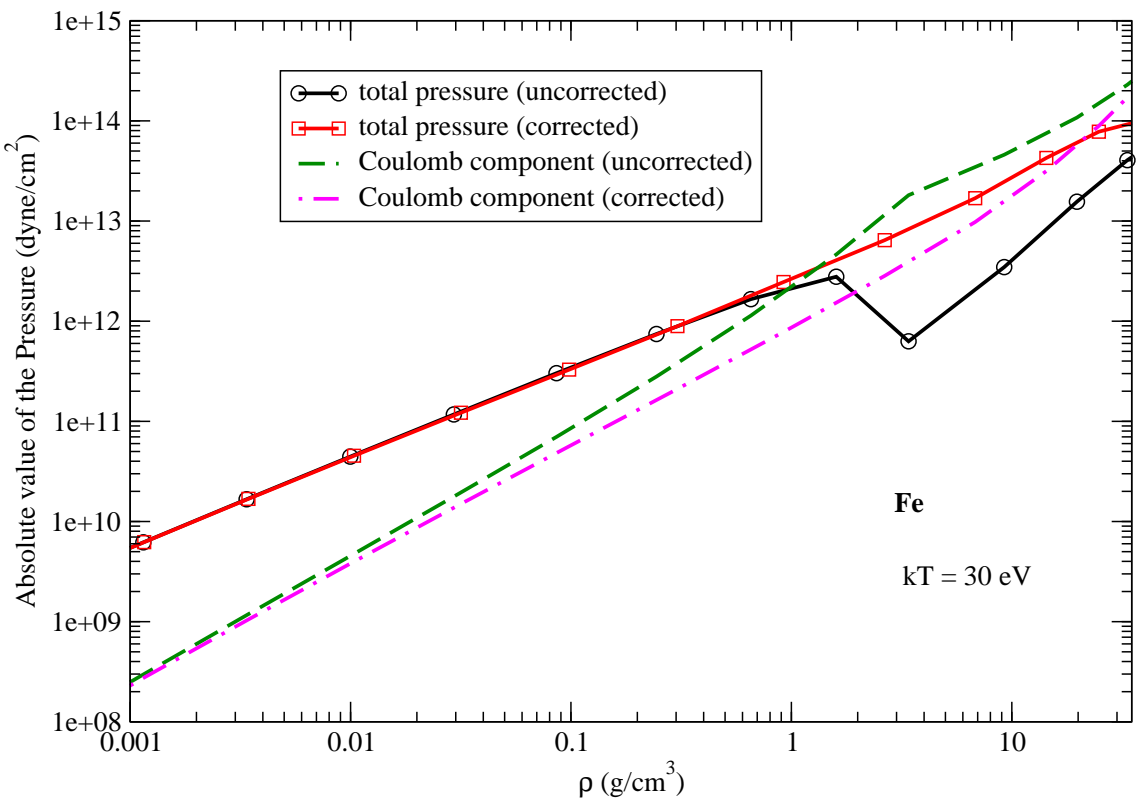

Figure 2: Total pressure $P$ and the absolute value of the Coulomb pressure component $\left|P_{C}\right|$ for the EOS without and with the correction to the free energy component $f_{i e}$. 


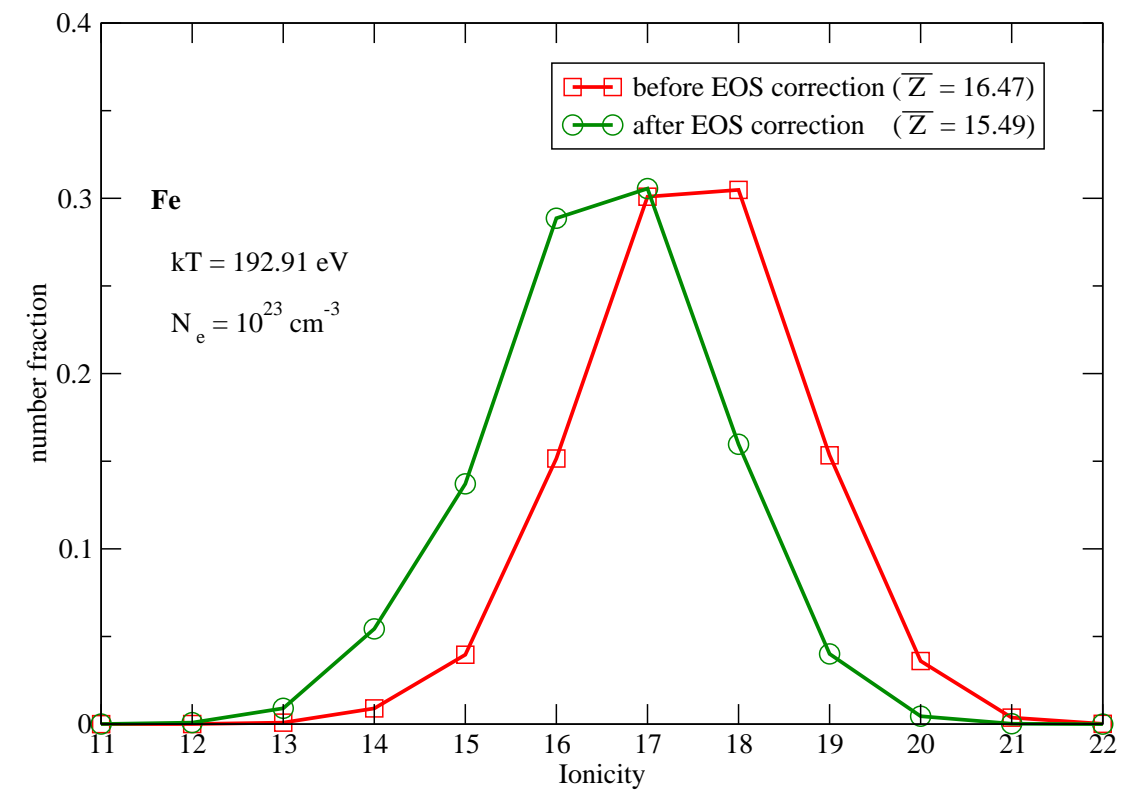

Figure 3: Charge state distribution of iron at the Sandia National Laboratory opacity experimental conditions. The different degrees of ionization arise from the EOS without (red squares) and with (green circles) the correction to the Coulomb interaction term. The corrected EOS leads to a less ionized plasma.

to 6.78 electrons from 6.32 electrons, a change of only about 7 percent and produces little change in the absorption between 8 and 9 Angstroms where there is still significant disagreement between theory and experiment.

\section{Conclusions}

We have adapted Chabrier's and Potekhin's Helmholtz free energy model [4] for the Coulomb interaction of plasma ions and electrons to approximately account for a mixture of ions of differing charge in the equation of state part of our LTE opacity calculations. We have further modified this term to approximately take into account the binding of electrons to the ions that is already accounted for in the internal partition function. The resultant equation of state quantities and average ionization show the absence of unphysical 


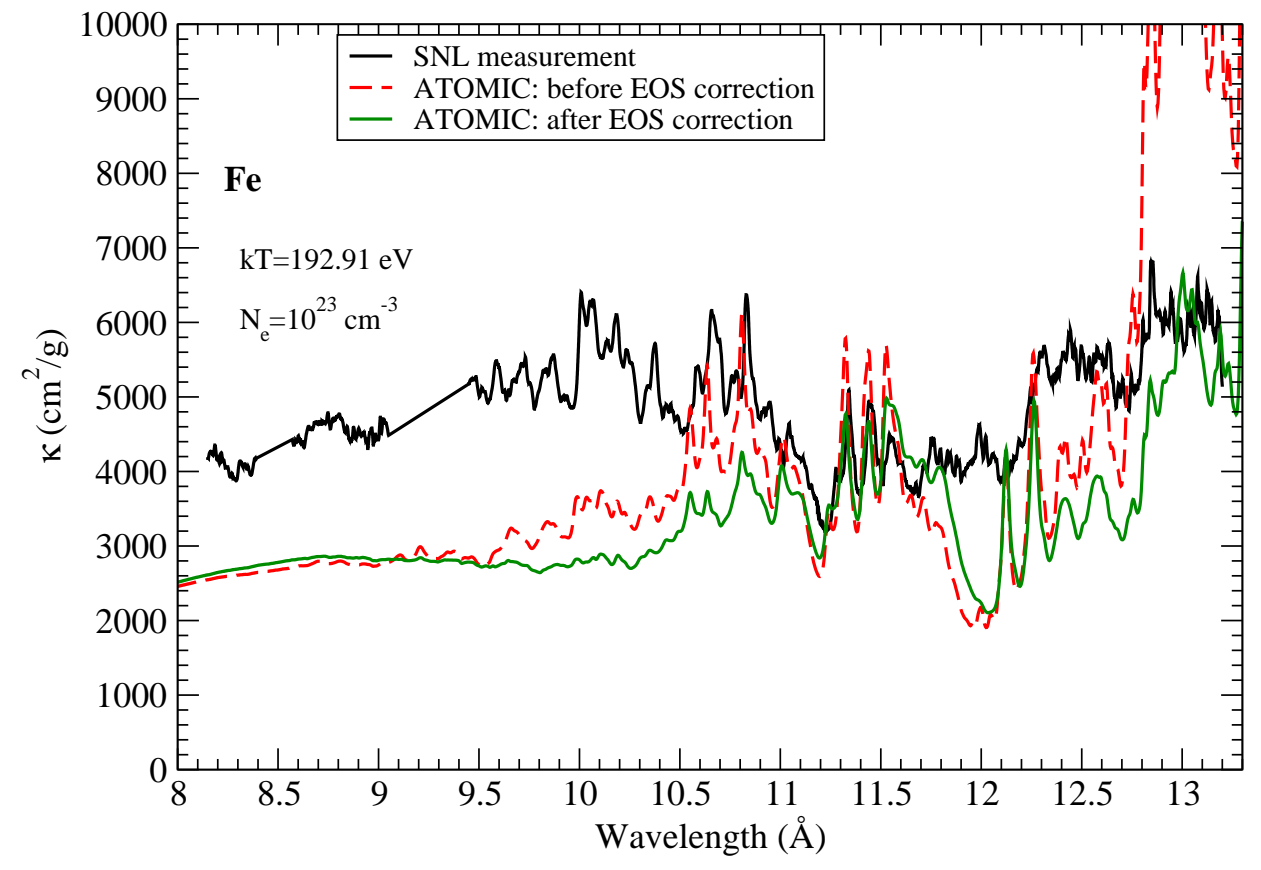

Figure 4: Sandia iron opacity experimental spectrum (black solid line) compared to Los Alamos ATOMIC calculations with (green solid line) and without (red dashed line) the corrected EOS. 
features present in the theory unmodified for these binding effects. The modified EOS is used in the Los Alamos opacity code ATOMIC to calculate the iron opacity relevant to recent opacity experimental measurements. Comparison of these calculations with the experiment show an improved agreement at longer photon wavelengths, but large disagreements still remain at shorter wavelengths.

\section{Acknowledgments}

The Los Alamos National Laboratory is operated by Los Alamos National Security, LLC for the National Nuclear Security Administration of the U.S. Department of Energy under Contract No. DEAC52-06NA25396.

\section{Appendix A. Definition of parameters}

For completeness we present definitions of the parameters appearing in the Coulomb interaction term $f_{i e}$ as given by Refs. $[4,8]$.

The electron coupling parameter is

$$
\Gamma_{e}=e^{2} /\left(k T a_{e}\right)=\frac{e^{2}}{k T}\left(\frac{4 \pi}{3} n_{e}\right)^{1 / 3} .
$$

The electron density parameter (electron sphere radius in Bohr radii) is

$$
r_{s}=a_{e} / a_{0}=\frac{1}{\left(\frac{4 \pi}{3} n_{e}\right)^{1 / 3}} \frac{m_{e} e^{2}}{\hbar^{2}} .
$$

The electron relativity parameter is

$$
x_{r}=p_{F} / m_{e} c=\frac{\hbar\left(3 \pi^{2} n_{e}\right)^{1 / 3}}{m_{e} c} .
$$

The Thomas-Fermi strong coupling limit is

$$
c_{T F}=\frac{18}{175}\left(\frac{12}{\pi}\right)^{2 / 3} Z^{7 / 3}\left(1-1 / Z^{1 / 3}+0.2 / Z^{1 / 2}\right) .
$$


Fitting functions dependent only on $Z$ are

$$
\begin{aligned}
a & =1.11 Z^{0.475} \\
b & =0.2+0.078(\ln Z)^{2} \\
\nu & =1.16+0.08 \ln Z .
\end{aligned}
$$

Fitting functions dependent only on the relativity parameter $x_{r}$ are

$$
\begin{gathered}
h_{1}\left(x_{r}\right)=\frac{1+x_{r}^{2} / 5}{1+0.18 Z^{-1 / 4} x_{r}+0.37 Z^{-1 / 2} x_{r}^{2}+x_{r}^{2} / 5}, \\
h_{2}\left(x_{r}\right)=\left(1+x_{r}^{2}\right)^{-1 / 2} .
\end{gathered}
$$

Additional fitting functions are

$$
\begin{gathered}
g_{1}=1+0.78\left(21+\Gamma_{e}\left(Z / r_{s}\right)^{3}\right)^{-1}\left(\Gamma_{e} / Z\right)^{1 / 2}, \\
g_{2}=1+\frac{Z-1}{9}\left(1+\frac{1}{0.001 Z^{2}+2 \Gamma_{e}}\right) \frac{r_{s}^{3}}{1+6 r_{s}^{2}} .
\end{gathered}
$$

Average kinetic energy for a free electron for any degeneracy is

$$
\bar{E}_{K E}=\frac{3}{2} k T \frac{I_{3 / 2}(\eta)}{I_{1 / 2}(\eta)} .
$$

The Debye-Hückel screening length is

$$
\lambda_{D}^{-1}=\sqrt{\frac{4 \pi e^{2}}{k T}\left(\sum_{s \neq e} n_{s} Z_{s}^{2}+n_{e} \Theta_{e}(\eta)\right)} .
$$

Note that in the limit of complete electron degeneracy $\eta \rightarrow \infty$ and $\Theta_{e}(\eta) \rightarrow 0$. However, the ratio $\Theta_{e} / k T=\eta \Theta_{e} / \mu_{e}$ remains finite because of the limit $\Theta_{e} \rightarrow(3 / 2) \eta^{-1}$ and $\mu_{e}$ becomes the electron Fermi energy $\mu_{e}(T=0)$ [10].

[1] P. Hakel, D. P. Kilcrease, in Atomic Processes in Plasmas, Eds., J. Cohen, S. Mazevet, D. Kilcrease, AIP, New York, 2004, p. 168.

[2] N. H. Magee, et al. in Atomic Processes in Plasmas, Eds., J. Cohen, S. Mazevet, D. Kilcrease, AIP, New York, 2004, p. 190. 
[3] P. Hakel, et al., J. Quant. Spectrosc. Rad. Transf. 99 (2006) 265.

[4] G. Chabrier, A. Y. Potekhin, Phys. Rev. E 58, (1998) 4941; A. Potekhin, G. Chabrier, Phys. Rev. E 62 (2000) 8554.

[5] A. Y. Potekhin, G. Chabrier, F. Rogers, Phys. Rev. E 79 (2009) 016411.

[6] G. M. Harris, J. E. Roberts, J. G. Trulio, Phys. Rev. 119 (1960) 1832.

[7] J. Colgan, et al., High Energy Density Phys. 14 (2015) 33.

[8] W. Däppen, D. Mihalas, D.G. Hummer, B. W. Mihalas, Astrophys. J. 332 (1988) 26.

[9] D. A. McQuarrie, Statistical Mechanics, Harper and Row, New York, 1976.

[10] D. P. Kilcrease, N. H. Magee, J. Quant. Spectrosc. Rad. Transf. 71 (2001) 445.

[11] D. Saumon, G. Chabrier, H. M. Van Horn, Astrophys. J. Sup. 99 (1995) 713.

[12] J. E. Bailey, et al., Nature 517 (2015) 56. 